\title{
Finite-size scaling and intermittency studies in crucial measurements for the QCD critical point
}

\author{
F. K. Diakonos* \\ Faculty of Physics, University of Athens, GR-15784, Greece \\ E-mail: fdiakono@phys.uoa.gr
}

\begin{abstract}
We propose a systematic way to search for the location of the QCD critical point in relativistic ion collisions, based on the relation of intermittency in momentum space to finite-size scaling (FSS) in configuration space. This relation is valid for systems equilibrating within the FSS region. The key ingredients of our method are combined measurements of the intermittency index $\phi_{2}$ in transverse momentum space of protons produced in central $\mathrm{A}+\mathrm{A}$ collisions and of the corresponding freeze-out parameters $\mu_{b}$ (baryochemical potential) and $T$ (temperature). We demonstrate how the proposed method works in practice using NA49 data for central Si+"Si" collisions at $\sqrt{s}=17.2 \mathrm{GeV}$. Based on these data we extract a set of predictions, concerning reachability of the critical endpoint, for the running NA61 experiment at SPS (CERN).
\end{abstract}

Corfu Summer Institute 2017 'School and Workshops on Elementary Particle Physics and Gravity' 2-28 September 2017

Corfu, Greece

\footnotetext{
* Speaker.
} 


\section{Introduction}

The exploration of the QCD phase diagram through ion collisions is a contemporary intensive experimental program [1,2] guided by a plethora of theoretical works focusing on the existence of a critical point at finite baryon density and temperature [3], remnant of the spontaneous breaking of chiral symmetry. Despite the intensive efforts, the search for the QCD critical point is up to now without success. An exception provides the intermittency analysis of dipion [4] and proton [5] transverse momenta in central $\mathrm{Si}+$ "Si" collisions at $\sqrt{s}=17.2 \mathrm{GeV}$ in NA49 experiment at SPS (CERN). There have been found indications of non-conventional (possibly critical) fluctuations, however, due to the large statistical and systematic errors, the confidence level in the drawn conclusions is quite low.

Searching for the QCD critical endpoint in ion collisions implies specific constraints for the procedure(s) which can be followed. Firstly, due to the finite size of the colliding nuclei the critical point, defined for an infinite system, becomes a critical region [6]. Secondly, the universality class of the transition restricts significantly the extension of the critical region. This is clearly shown in [7], where, employing a suitable partition function (Ising-QCD), the size of the critical region along the chemical potential direction is estimated to be of the order of a few $(\approx 5) \mathrm{MeV}$. As discussed in $[7,8]$, this extreme narrowness of the critical region has significant consequences on its reachability. In particular, the beam energy scan in STAR experiment (RHIC, BES I) cannot achieve freeze-out states entering into the critical region, since in this protocol the chemical potential varies in steps of $\approx 50 \mathrm{MeV}$. A better strategy to enter into the critical region is to use ions of a medium size $20<A<50$ colliding with beam energy $\sqrt{s}$ in the range $16-18 \mathrm{GeV}$. These conditions could, in principle, be met at the NA61 experiment (SPS, CERN). A third constraint comes from the fact that the benchmark of the critical region is the scaling of the density-density correlation function of the order parameter (proton density, sigma condensate) in configuration space. In ion collisions there is no direct access to information concerning configuration space. Thus, one has to look for traces of this scaling behaviour in momentum space. The solution to this task is provided by the finite size scaling (FSS) theory of critical phenomena. Namely, within the FSS region, which is a subregion of the critical region, the scaling holds for arbitrarily large spatial scales: the upper limit is set by the correlation length of the infinite system and not by the system's size. This means, as it can be shown by an appropriate Fourier transform [9], that the spatial scaling is transferred to a scaling of the density-density correlation function in momentum space for arbitrarily small momentum differences [10]. Arguments related to the geometry of the collision imply that the appropriate space to look for this scaling is the transverse momentum space [11].

In the current work we demonstrate that accurate measurements of the intermittency index $\phi_{2}$ through proton transverse momentum intermittency analysis in central, medium size $(20<A<50)$ nuclei collisions at maximum SPS energy $(\sqrt{s} \approx 17 \mathrm{GeV})$, when combined with accurate measurements of the freeze-out chemical potential $\left(\mu_{b}\right)$ and temperature $(T)$, can lead to the determination of the critical point location. To achieve this, we use the published central (most probable) values for the intermittency index $\phi_{2}$ [5] and for the corresponding freeze-out parameters [12], as measured in central $\mathrm{Si}+$ "Si" collisions at $\sqrt{s}=17.2 \mathrm{GeV}$ (NA49 experiment).

The work is organized as follows. In section II we estimate the extension of the FSS region for a set of nuclei. In section III we propose an interpolating scheme allowing us to determine 
the dependence of the freeze-out parameter values $\left(\mu_{b}, T\right)$ on the mass number $A$ for fixed beam energy $\sqrt{s}=17.2 \mathrm{GeV}$. To achieve this we use freeze-out data measured in NA49 experiment [12]. In section IV we develop a procedure which allows us to determine the location of the QCD critical point using $\left(\mu_{b}, T, \phi_{2}\right)$-values for a system freezing out within the FSS region. Finally, in section $\mathrm{V}$ we briefly present our conclusions.

\section{Estimating the size of the FSS region}

We will adopt here the conjecture that the QCD critical point belongs to the $3 \mathrm{~d}$ Ising universality class. As discussed in the introduction, the extension of the critical region along the chemical potential direction is determined by the universality class [7]. The FFS property provides constraints along the temperature direction. Since within the critical region there are no distinguishable phases of the critical fluid, one has to consider both an upper $\left(T_{+}>T_{c}\right)$ and a lower $\left(T_{-}<T_{c}\right)$ limit of the FSS region ( $T_{c}$ being the critical temperature). Let us define $t=\left|\frac{T-T_{c}}{T_{c}}\right|$ with $T$ the freeze-out temperature. Then, the correlation length of the infinite system $\xi_{\text {inf }}$ within the FSS region is given by:

$$
\xi_{\text {inf }}(T)=\xi_{0} t^{-v} \quad ; \quad v=\frac{2}{3} \quad(3 \mathrm{~d} \text { Ising }) .
$$

with $\xi_{0}=\xi_{0,+}$ (for $T>T_{c}$ ) or $\xi_{0}=\xi_{0,-}$ (for $T<T_{c}$ ). The coefficients $\xi_{0, \pm}$ are non-universal but they possess a universal ratio $\frac{\xi_{0,+}}{\xi_{0,-}}=2$. By definition the border of the FSS region is given as:

$$
\xi_{\text {inf }}\left(T_{ \pm}\right)=R \quad \Rightarrow \quad t_{ \pm}=\left(\frac{\xi_{0, \pm}}{L}\right)^{1 / v}
$$

where we introduced the notation $t_{ \pm}=\left|\frac{T_{ \pm}-T_{c}}{T_{C}}\right|$ and $L$ is the system's size. As already mentioned, for the fireball geometry emerging in ion collisions, we are interested for the transverse size $L_{\perp}$ estimated to be $L_{\perp}=\sqrt{S}$, with $S=\pi R^{2}$ the fireball's average transverse area. The corresponding radius is $R=R_{0} A^{1 / 3}$ with $R_{0} \approx 1.25 \mathrm{fm}$. Clearly to obtain $T_{ \pm}$we need to know $T_{c}$ and $\xi_{0,+}$ (or $\xi_{0,-}$ ). Here we will use $T_{c}=164 \mathrm{MeV}$, as supported by recent Lattice QCD calculations [13], and $\xi_{0,+}=\frac{1}{\Lambda_{Q C D}}$ which is a natural scale for strong interaction physics. Using recent estimates $\Lambda_{Q C D} \approx 260 \mathrm{MeV}$ [14] we get $\xi_{0,+}=0.75 \mathrm{fm}$. With this choice and using Eq. (2.2) we obtain the results for the FSS region limits shown in Table I:

\section{Freeze-out states at maximum SPS energy}

Our next step is to calculate the freeze-out chemical potential and temperature values for different A+A systems colliding with maximum SPS energy $\sqrt{s}=17.2 \mathrm{GeV}$. To achieve this, we can use the mean values of the freeze-out parameters of the fireball formed in central $\mathrm{C}+\mathrm{C}, \mathrm{Si}+\mathrm{Si}$, $\mathrm{Pb}+\mathrm{Pb}$ collisions at this beam energy, as estimated in [12]. Our aim is to obtain smooth interpolating curves for $T=T(A)$ and $\mu_{b}=\mu_{b}(A)$ which are monotonic. It turns out that the function $T=T(A)$ is well described by the relation:

$$
T=T_{0}+T_{a} A^{-w_{T}} \quad ; \quad T_{0}=154.56 \mathrm{MeV}, T_{a}=37.39 \mathrm{MeV}, w_{T}=0.48
$$




\begin{tabular}{|l|c|c|}
\hline System $(\mathrm{A})$ & $T_{+}(\mathrm{MeV})$ & $T_{-}(\mathrm{MeV})$ \\
\hline \hline $\mathrm{Be}(8)$ & 175.60 & 159.90 \\
\hline $\mathrm{C}(12)$ & 173.47 & 160.65 \\
\hline $\mathrm{Si}(28)$ & 170.20 & 161.81 \\
\hline $\mathrm{Ar}(40)$ & 169.19 & 162.17 \\
\hline $\mathrm{Sc}(45)$ & 168.89 & 162.27 \\
\hline $\mathrm{Xe}(131)$ & 166.87 & 162.99 \\
\hline $\mathrm{La} \mathrm{(139)}$ & 166.78 & 163.02 \\
\hline $\mathrm{Au}(197)$ & 166.34 & 163.17 \\
\hline $\mathrm{Pb}(208)$ & 166.27 & 163.20 \\
\hline
\end{tabular}

Table 1: Estimated limits for the FSS region according to the description in eq. (2.2).

while for the function $\mu=\mu(A)$ we obtain:

$$
\mu_{b}=\mu_{0}+\mu_{a} e^{-w_{\mu} A} \quad ; \quad \mu_{0}=247.36 \mathrm{MeV}, \mu_{a}=17.54 \mathrm{MeV}, w_{\mu}=0.01
$$

The resulting functions are presented graphically in Fig. 1.

\section{Locating the critical point}

As shown in [8], employing the Ising-QCD partition function, all the freeze-out states with constant intermittency index $\phi_{2}$ define an (approximately) piecewise linear line in the $(\ln \zeta, t)$ plane with $\ln \zeta=\frac{\mu_{b}-\mu_{c}}{k_{B} T_{c}}, t=\frac{T-T_{c}}{T_{c}}$ and $\mu_{c}$ the (unknown) critical chemical potential. This line can be determined by a linear fit in each of its branches obtaining this way a set of two linear relations (for $t>0$ and $t<0$ respectively) between $t$ and $\ln \zeta$. Subsequently, one can use this linear correlation to determine the critical chemical potential $\mu_{c}$ in terms of the critical temperature $T_{c}$, provided that a triplet $\left(\mu_{b}, T, \phi_{2}\right)$, within the critical region, is known. In fact, this information, as already mentioned, is available for the freeze-out state formed in central $\mathrm{Si}+$ "Si" collisions at highest SPS energy, being measured in the NA49 experiment. For simplicity, since we are mainly interested to demonstrate how the proposed procedure works in principle, we proceed ignoring the (quit large) experimental errors. The line for which $\phi_{2} \approx 0.96$ (blue line in Fig. 2) is very well approximated by the piecewise linear function:

$$
t^{(S i)}=a_{ \pm}^{(S i)}+b_{ \pm}^{(S i)} \ln \zeta^{(S i)}
$$

with $a_{+}^{(S i)} \approx-0.51, b_{+}^{(S i)} \approx 41.19$ (valid for $t>0$ ) and $a_{-}^{(S i)} \approx 0.17, b_{-}^{(S i)} \approx-14.09$ (valid for $t<0$ ). Equation (4.1) implies a linear relation between $\mu_{c}$ and $T_{c}$ which depends on the sign of $t^{(S i)}$ :

$$
\mu_{c}= \begin{cases}256.06 \mathrm{MeV}+0.01 T_{c}, & T^{(S i)}>T_{c} \\ 271.51 \mathrm{MeV}-0.08 T_{c}, & T^{(S i)}<T_{c}\end{cases}
$$

with $T^{(S i)}$ the temperature of the $\mathrm{Si}+$ "Si" freeze-out state. Using $T^{(S i)}=162.2 \mathrm{MeV}$ [12] and $T_{c}=164 \mathrm{MeV}$ [13] we find $\mu_{c}=257.7 \mathrm{MeV}$. Using this value and the fit results of the previous 

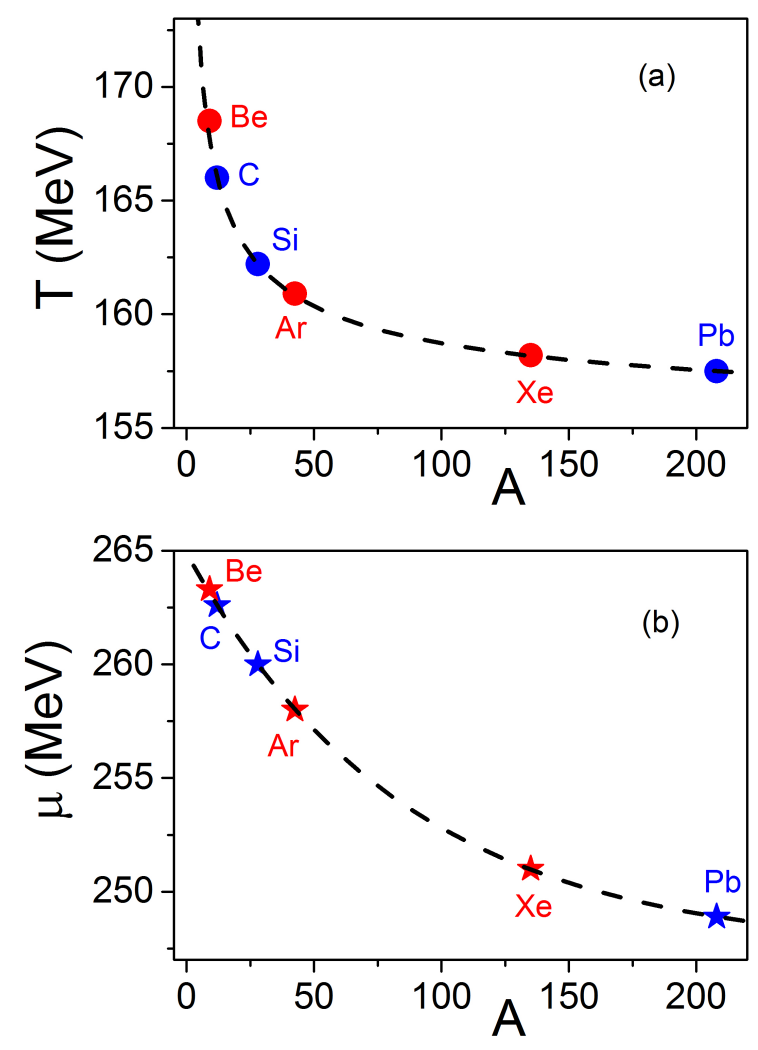

Figure 1: (a) The temperature of the freeze-out states for central $A+A$ collisions in NA49 experiment at $\sqrt{s}=17.2 \mathrm{GeV}$ according to [12] are given by the blue circles. The red circles are predictions for the freeze-out temperature of central A+A' collisions in NA61 experiment $(\sqrt{s}=17.2 \mathrm{GeV})$ estimated using eq. (3.1) (black solid line). (b) Similar to (a) but now for the freeze-out chemical potential. The existing data for NA49 A+A collisions [12] are shown by the blue stars while the red stars are predictions for the A+A' collisions in NA61/SHINE experiment. The black solid line represents the graph of eq. (3.2).

section we can make a set of predictions for the freeze-out states expected to be measured in the NA61 experiment. The results are summarized in Fig. 2. We observe that the $\mathrm{Si}+$ "Si" freezes out within the FSS region. The Ar + Sc system freezes out within the critical region but outside the FSS region and therefore it is expected to show some scaling, however not at arbitrary small transverse momentum scales. The freeze-out states of the $\mathrm{Be}+\mathrm{Be}, \mathrm{Xe}+\mathrm{La}$ and $\mathrm{Pb}+\mathrm{Pb}$ systems lie out of the critical region. No freeze-out state of Au + Au system in STAR (RHIC) can fit in this plot.

\section{Conclusions}

We propose a method for locating the QCD critical point using accurate measurements of the triplet $\left(\mu_{b}, T, \phi_{2}\right)$ in $\mathrm{A}+\mathrm{A}$ " collisions. The basic requirement is that the corresponding freeze-out state lies within the FSS region, a condition which is fulfilled for the state formed in central $\mathrm{Si}+$ 


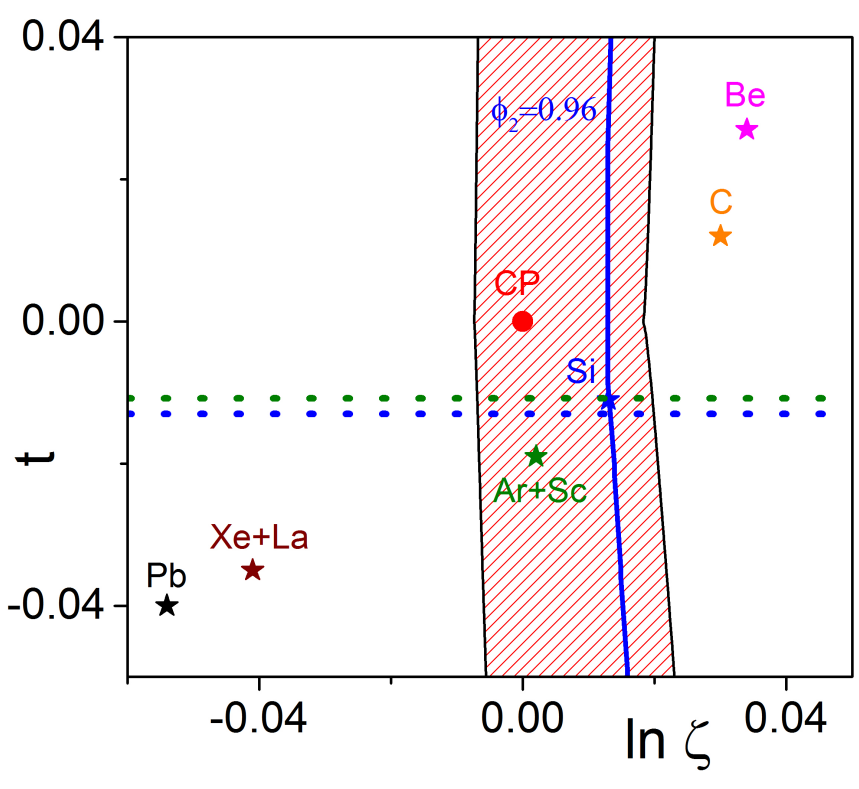

Figure 2: The critical region (red shaded area) in the $(\ln \zeta, t)$ plane determined using the Ising-QCD partition function [8,10] and assuming $T_{c} \approx 164 \mathrm{MeV}$. The colored points indicate the location of the different freezeout states measured in NA49 experiment [12] or expected to be measured in the NA61 experiment. The blue line is the graphical presentation of $\phi_{2}=0.96$ passing through the freeze-out state of $\mathrm{Si}+\mathrm{Si}$ ". In general the errors (not shown here), especially for the freeze-out chemical potential, are very large and more accurate measurements are needed. The dotted lines (blue, green) present the boarders of the FSS region in each case $(\mathrm{Si}+\mathrm{Si} ", \mathrm{Ar}+\mathrm{Sc})$.

"Si" collisions at maximum SPS energy $(\sqrt{s}=17.2 \mathrm{GeV})$. Our analysis indicates that the search for the critical point should be performed using medium size nuclei $(20<A<40)$ colliding at energies close to $\sqrt{s}=17 \mathrm{GeV}$.

\section{References}

[1] M. Gazdzicki (for the NA61 Collaboration), J. Phys. G: Nucl. Part. Phys. 36, 064039 (2009); N. Antoniou et al. (NA61/SHINE Collaboration), CERN-SPSC-2006-034.

[2] M. M. Aggarwal et al. (STAR Collaboration), arXiv:1007.2613; STAR Note 0598: BES-II whitepaper: http://drupal.star.bnl.gov/STAR/starnotes/public/sn0598.

[3] S. Gavin, A. Gocksch and R. D. Pisarski, Phys. Rev. D 49, 3079 (1994); M. Stephanov, K. Rajagopal and E. Shuryak, Phys. Rev. Lett. 81, 4816 (1998); M. A. Halasz, A. D. Jackson, R. E. Shrock, M. A. Stephanov and J. J. M. Verbaarschot, Phys. Rev. D 58, 096007 (1998); J. Berges and K. Rajagopal, Nucl. Phys. B 538, 215 (1999); F. Karsch, E. Laermann and Ch. Schmidt, Phys. Lett. B 520, 41 (2001).

[4] T. Anticic et al., Phys. Rev. C 81, 064907 (2010).

[5] T. Anticic et al., Eur. Phys. J. C 75, 587 (2015). 
[6] R. V. Gavai, "The QCD critical point: an exciting Odyssey in the Femto-world", Contemporary Physics 57, 350 (2016).

[7] N. G. Antoniou, F. K. Diakonos, N. Kalntis and A. Kanargias, arXiv:1711.10315 [nucl-th].

[8] N. G. Antoniou, F. K. Diakonos, X. N. Maintas and C. E. Tsagkarakis, Phys. Rev. D 97, 034015 (2018).

[9] N. G. Antoniou, N. Davis and F. K. Diakonos, Phys. Rev. C 93, 014908 (2016).

[10] See the contribution of N. G. Antoniou in this proceedings.

[11] N. G. Antoniou, F. K. Diakonos, A. S. Kapoyannis and K. S. Kousouris, Phys. Rev. Lett. 97, 032002 (2006).

[12] F. Becattini, J. Manninen and M. Gazdzicki, Phys. Rev. C 73, 044905 (2006).

[13] S. Datta, R. V. Gavai and S. Gupta, Phys. Rev. D 95, 054512 (2017).

[14] J.-L. Kneur and A. Neveu, Phys. Rev. D 85, 014005 (2011). 